\title{
CHARACTERISTICS OF BURDEN RESISTORS FOR HIGH-PRECISION DC CURRENT TRANSDUCERS
}

\author{
G. Fernqvist, P. Dreesen, G. Hudson, J. Pickering ${ }^{1)}$ \\ CERN, Geneva, Switzerland
}

\begin{abstract}
The DC current transducer (DCCT) and accompanying A/D converter determine the precision of a power converter in accelerator operation. In the LHC context this precision approaches $10^{-6}(1 \mathrm{ppm})$. Inside the DCCT a burden resistor is used to convert the current to an output voltage. The performance of this resistor is crucial for the accuracy, temperature behaviour, settling time and longterm drift of the DCCT. This paper reports on evaluations, a new parameter called "power coefficient" (PC) and test results from some different types of resistors available on the market.
\end{abstract}

${ }^{1)}$ Metron Designs, Norwich, UK

CERN, 


\title{
CHARACTERISTICS OF BURDEN RESISTORS FOR HIGH-PRECISION DC CURRENT TRANSDUCERS
}

\author{
G. Fernqvist, P. Dreesen, G. Hudson, CERN, Geneva, Switzerland \\ J. Pickering, Metron Designs, Norwich, UK
}

\section{Abstract}

The DC current transducer (DCCT) and accompanying $\mathrm{A} / \mathrm{D}$ converter determine the precision of a power converter in accelerator operation. In the LHC context this precision approaches $10^{-6}(1 \mathrm{ppm})$. Inside the DCCT a burden resistor is used to convert the current to an output voltage. The performance of this resistor is crucial for the accuracy, temperature behaviour, settling time and longterm drift of the DCCT. This paper reports on evaluations, a new parameter called "power coefficient" (PC) and test results from some different types of resistors available on the market.

\section{INTRODUCTION}

The magnet current in accelerators has always required a high precision to maintain the beams in position and well focused. The power converters supplying this current use a precision current transducer in a feedback loop to control the output current. For high precision and high current a precision transducer with galvanic isolation is the preferred choice. The dominant technology is a zeroflux current transformer, which is a transformer in an active feedback loop with the bandwidth extending down to DC. This configuration [1] can divide the primary current with an arbitrary factor to a manageable small current $(100 \mathrm{~mA}-10 \mathrm{~A})$ with a ratio error $<1 \mathrm{ppm}$ (part per million, $\left.10^{-6}\right)$.

The output of the transformer is connected to a burden, a 2-terminal or 4-terminal resistor depending on the resistor value and the required precision. The performance of this component is one of the dominant factors in the overall precision of the transducer.

Previous accelerators have in general required reproducibility from the transducer approaching $10 \mathrm{ppm}$ and an accuracy of $100 \mathrm{ppm}$. The LHC collider [2] requires an improvement in the precision of more than an order of magnitude for many reasons, a very important one being the tracking between the 8 machine sectors. It was therefore necessary to examine the limitations and possible improvements of existing burden resistor design as part of the LHC development programme.

\section{EARLY EXPERIENCE}

A small number of DCCTs had been ordered for LHC prototypes and also a much larger quantity for the renovation of the SPS accelerator. The reception tests for these DCCTs were used to improve measurement infrastructure, techniques and methodology. Several parameters emerged as key points for a complete characterisation, i.e. temperature coefficient (TC), settling behaviour, linearity, short- and long-term drift, all originating in the burden resistor performance. The following specifications were applicable:

$\begin{array}{ll}\text { Long-term drift } & <5 \mathrm{ppm} / \text { year } \\ \text { Non-linearity } & <6 \mathrm{ppm} \\ \text { Temp. coeff. (TC) } & <1 \mathrm{ppm} / \mathrm{K}\end{array}$

The drift of several DCCTs using burden resistors with Zeranin foil was monitored over a year and is depicted in fig. 1. The large variations occurred with changes in operating conditions. Problems from humidity influence were also identified, but will not be dealt with in this paper. These discouraging observations initiated a very long investigation.

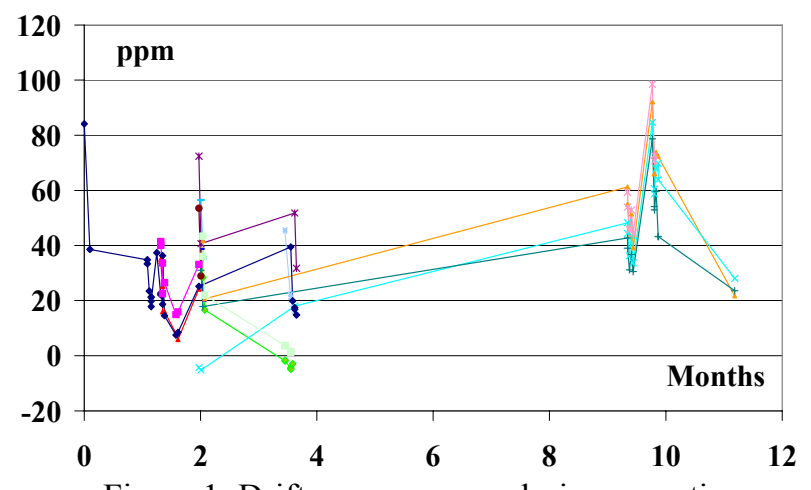

Figure 1: Drift over one year during operation.

\section{BURDEN RESISTOR CONSTRUCTION}

\section{Choice of Resistor Types}

The LHC applications require performance in the above mentioned characteristics at the ppm level. The highest precision is offered by a proprietary Zeranin wire design from Hitec, but its price limits the use to the best DCCTs. There is only one resistor type on the market offering the performance needed, Bulk Metal Foil or "foil". This technique, pioneered by Vishay, tightly bonds a rolled metal foil to a substrate and seeks to compensate the resulting consistent stress effects as part of the overall resistor performance. Only this technology is considered in the descriptions below. A metal can was generally specified to avoid humidity influence.

It is not enough to think of precision burden resistors, which have to dissipate some power, as components whose performance can be calculated from the TC and the temperature rise due to the dissipated power (Power • Thermal Resistance, the latter called $\theta \mathrm{a}$ and typically in $\mathrm{K} /$ Watt). In practice any resistor's performance is made up from a combination of the resistive element's (foil or wire) stability and its thermal and mechanical packaging and mounting. 


\section{Power Coefficient of Resistance (PC)}

As the power dissipated in a resistor increases, its temperature will rise and its resistance will change as a result. However, it was soon found that, even with near zero TC, the resistance value of a typical burden changes with applied current, causing a non-linear measurement of current. It was deduced that this is due to the "flow" of heat from the dissipating foil to the substrate, which gives a temperature difference between foil and substrate independent of changes in ambient temperature. In other words, the stress balance inherent in this type of resistor is upset and this can have both TC and long-term effects on the resistance value.

Fig. 2 shows a simple cross sectional view of a Bulk Metal Foil current sense resistor. The design aims to achieve high stability whilst taking advantage of its planar construction to be heat-sunk.

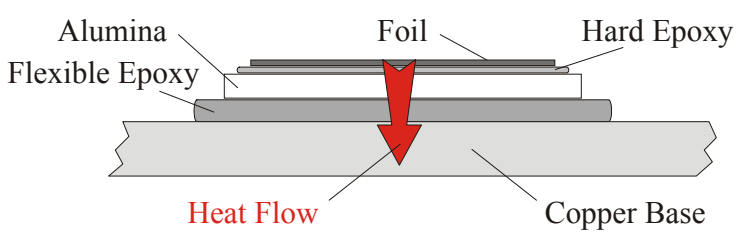

Figure 2: Foil current sense resistor construction.

The foil/hard-epoxy/alumina-substrate combination is designed to give zero $\mathrm{TC}$ to ambient changes of temperature, i.e. to changes that affect all the layers equally. The resistance TC of the foil is generally +10 to $+20 \mathrm{ppm} / \mathrm{K}$ and, because it has a greater coefficient of thermal expansion than the alumina, it is restricted by the tight bonding which then pulls the TC negative by the opposite amount when both are heated equally. If the foil alone was heated, the effect of its mechanical expansion relative to the alumina's non-expansion would be even greater, pulling the $\mathrm{TC}$ too far negative. This is what happens when the resistor is dissipating power: the foil heats up more than the substrate and is "over-restricted", causing its resistance to decrease. If the thermal resistance of the foil to substrate is $\theta_{\mathrm{fs}}(\mathrm{K} / \mathrm{W})$, a resistance change can be expected, when dissipating power, $\mathrm{P}$, of:

$$
\Delta \mathrm{R} / \mathrm{R}=-\mathrm{TC}_{\text {foil }} \bullet \theta_{\mathrm{fs}} \cdot \mathrm{P} \text { or } \mathrm{PC} \cdot \mathrm{P}(\mathrm{ppm})
$$

The reason for " $-\mathrm{TC}_{\text {foil }}$ " being that this, for a zero $\mathrm{TC}$ resistor, must be the restricting effect of the substrate.

Thus there are three major factors: resistance change due to ambient temperature (TC), the bulk self-heating effect of $\mathrm{P} \bullet \theta_{\mathrm{a}} \cdot \mathrm{TC}$ and the new "true" power coefficient of resistance ( $\mathrm{PC}=-\mathrm{TC}_{\mathrm{foil}} \cdot \theta_{\mathrm{fs}}$ ) or the change due to the chip/carrier gradient, in other words the PC of a zero TC resistor. In practice, where one manufacturer does specify a sort of PC, he includes self-heating:

$$
\mathrm{WCR}=\left(\mathrm{TC} \bullet \theta_{\mathrm{a}}\right)+\left(-\mathrm{TC}_{\mathrm{foil}} \cdot \theta_{\mathrm{fs}}\right), \quad(\mathrm{ppm} / \text { Watt })
$$

Note that this could be zero for quite large individual factors, so, for our purpose, less useful.

\section{Implications for Settling Time and Stability}

It has been shown that under steady power conditions the stress between foil and substrate is different from that when no power is dissipated and that this can be quite different from that due to ambient temperature changes. There is also evidence in the measurements that the effect of this stress is partially dependent on time, causing "memory-like" long-term settling tails and long-term recovery, presumably as the induced stresses slowly relax. This can take from minutes to days and is of great significance at the highest precision levels.

\section{MEASURING EQUIPMENT}

Two main methods are used at CERN to measure the resistance and its variation. Both methods are fully automated with computer control.

The first is based on a resistance bridge, MIL 6010B with a 100A extender 6011 and is dependent on calibrated standard resistors in a temperature controlled oil bath. The accuracy and resolution are sub-ppm.

The second is based on the CERN DCCT calibrator (CDC) [3], which produces a 24-bit programmable DC current up to $10 \mathrm{~A}$ with an accuracy of $<1 \mathrm{ppm}$, and an Agilent 3458 DVM.

\section{RESULTS FROM LHC PRODUCTION}

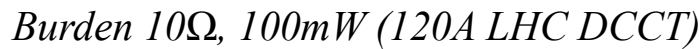

Settling behaviour and drift problems were quickly observed and several resistors were then measured whilst cycling power and temperature to verify if artificial aging would improve performance. The results were not coherent and indicated an unpredictable behaviour, see fig. 3 .

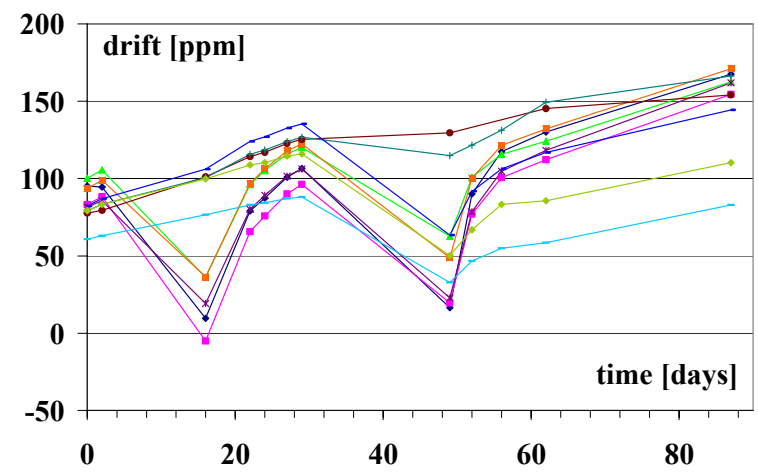

Figure 3: Drift with changing operating conditions.

The resistors were replaced with a different type by the manufacturer and the stability over days for the new resistors was then acceptable, but the $30 \mathrm{~min}$ stability criterion $(<10 \mathrm{ppm})$ resulted in $>10 \%$ rejection, see fig. 4 . The initial step comes from the power coefficient and the drift over 5-10 min comes from the self-heating. 


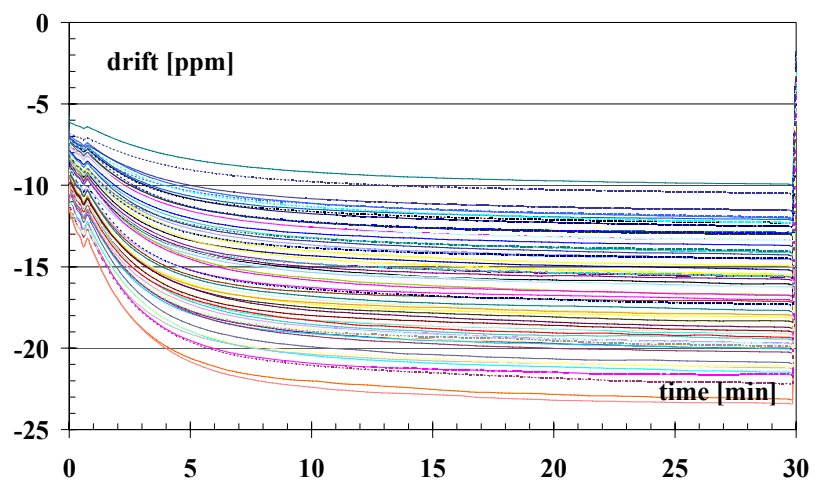

Figure 4: Stability over 30 min after applying nominal I

As the replacement of bad resistors on the PCB was very time-consuming, the resistors were screened before mounting by measuring the error caused by self-heating and that by the PC. Fig. 5 shows an interesting correlation between the two parameters. A different optimisation between the two can perhaps be made by the manufacturer, depending on the application. The results are based on over 700 resistors supplied by Alpha and Vishay.

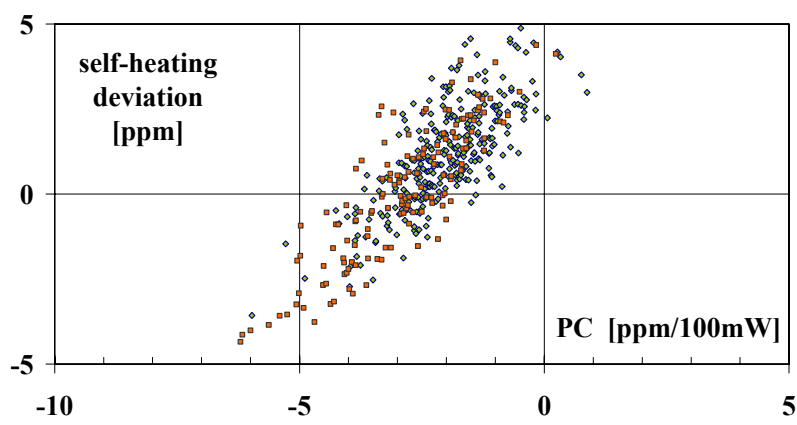

Figure 5: Self-heating error vs. PC of LHC $10 \Omega$ chip resistors.

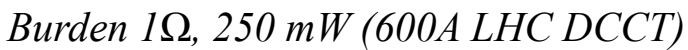

The $1 \Omega$ burden was developed by Vishay for LHC and the results from the pre-series are shown in figs 6 and 7.

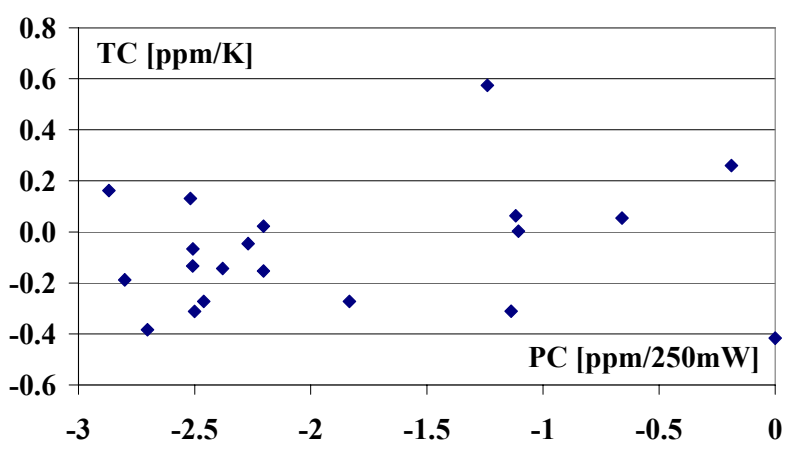

Figure 6: TC vs. PC of LHC $1 \Omega$ chip resistors.

No strong correlation between TC and PC can be seen for this small sample (20). The long-term drift is around $10 \mathrm{ppm} / \mathrm{year}$ and will likely decrease with time. As the tests of the complete DCCTs were satisfactory, no further reception tests were done on these resistors.

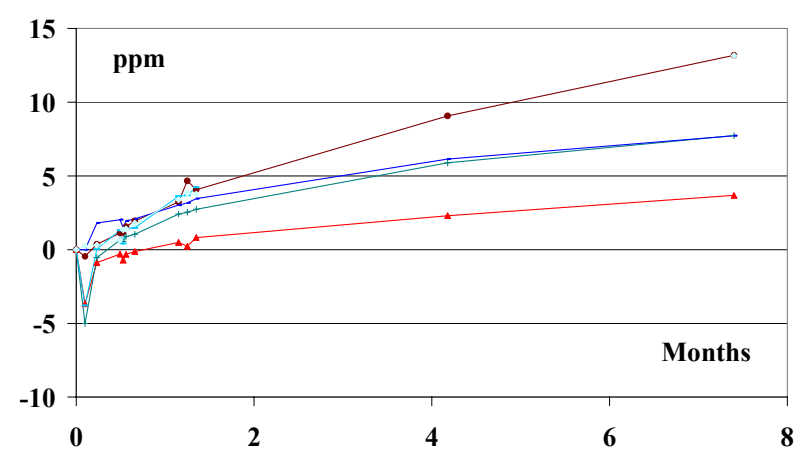

Figure 7: Long term drift of LHC $1 \Omega$ chip resistors

\section{CONCLUSIONS}

- Resistor performance with variable internal power dissipation is rarely given in data sheets, but needs consideration to obtain high accuracy.

- The TC of a resistor will directly impact its stability and accuracy due to ambient temperature variations and this can easily be measured. Modern chipresistors can be better than $1 \mathrm{ppm} / \mathrm{K}$.

- Self-heating of a resistor from the internal power dissipation will cause a resistance change due to the $\mathrm{TC}$, but this change can be made arbitrarily small by decreasing the dissipation per resistor. Its effect is a time-dependent non-linearity, governed by mounting method, cooling etc.

- The PC is a different effect from self-heating and almost instantaneous. It will cause a timeindependent, i.e. reproducible, non-linearity. In most accelerator applications this non-linearity is acceptable, because reproducibility is more important than absolute accuracy. Vishay is now specifying a kind of PC, but has chosen to include the effect of self-heating in this parameter.

- Burden resistors may have a slow drift of tens of ppms over a period of days to weeks depending on operating conditions. This effect is probably due to slow stress changes in the resin from temperature variations.

\section{REFERENCES}

[1] H. Appelo, M. Groenenboom, J. Lisser, The zeroflux DC current transformer, IEEE Trans. Nucl. Science, vol. NS-24, no 3, June 1977

[2] LHC Design Report, CERN-2004-003, Geneva

[3] G. Fernqvist et al, "A novel current calibration system up to 20kA", IEEE Trans. Instrum. Meas, vol. 52, April 2003 